University of Wollongong

Research Online

Faculty of Engineering and Information

Faculty of Engineering and Information

Sciences - Papers: Part A

Sciences

$1-1-2015$

\title{
Analysis of a chemostat model with variable yield coefficient and substrate inhibition: contois growth kinetics
}

Rubayyi Turki Alqahtani

University of Wollongong, rtaa648@uowmail.edu.au

Mark I. Nelson

University of Wollongong, mnelson@uow.edu.au

Annette L. Worthy

University of Wollongong, annie@uow.edu.au

Follow this and additional works at: https://ro.uow.edu.au/eispapers

Part of the Engineering Commons, and the Science and Technology Studies Commons

Research Online is the open access institutional repository for the University of Wollongong. For further information contact the UOW Library: research-pubs@uow.edu.au 


\title{
Analysis of a chemostat model with variable yield coefficient and substrate inhibition: contois growth kinetics
}

\author{
Abstract \\ 2015, Copyright Taylor \& Francis Group, LLC. We analyze the steady-state operation of a generalized \\ reactor model that encompasses a continuous flow bioreactor and an idealized continuous flow \\ membrane reactor as limiting cases. The biochemical reaction kinetics is governed by a Contois growth \\ model subject to noncompetitive substrate inhibition with a variable substrate yield coefficient. The \\ steady-state performance of the reactor is predicted and stability of the steady-state solutions as a \\ function of dimensionless residence time reported. Our results identified two cases of practical interest. \\ The first feature corresponds to the case where solutions to both no-washout and washout conditions are \\ bistable. The second feature identifies the parameter region in which periodic solutions can occur when \\ the yield coefficient is not constant. Both these features are often undesirable in practical applications \\ and must be avoided. Scaling of the model equations reveals that both the second-bifurcation parameters \\ are functions of the influent concentration. Our results predict how the reactor behavior varies as a \\ function of influent concentration and identify the range of influent concentrations where the reactor \\ displays neither periodic nor bistable behavior.
}

\section{Keywords}

substrate, coefficient, yield, variable, inhibition, model, analysis, chemostat, contois, growth, kinetics

\section{Disciplines}

Engineering | Science and Technology Studies

\section{Publication Details}

Alqahtani, R., Nelson, M. I. \& Worthy, A. L. (2015). Analysis of a chemostat model with variable yield coefficient and substrate inhibition: contois growth kinetics. Chemical Engineering Communications, 202 (3), 332-344. 


\title{
Analysis of a chemostat model with variable yield coefficient and substrate inhibition: Contois growth kinetics
}

\author{
Rubayyi T. Alqahtani Mark I. Nelson Annette L. Worthy \\ School of Mathematics and Applied Statistics, \\ University of Wollongong, NSW 2522, Australia
}

November 10, 2015

\begin{abstract}
We analyze the steady-state operation of a generalized reactor model that encompasses a continuous flow bioreactor and an idealised continuous flow membrane reactor as limiting cases. The biochemical reaction kinetics is governed by Contois growth model subject to noncompetitive substrate inhibition with a variable substrate yield coefficient. The steadystate performance of the reactor is predicted and stability of the steady-state solutions as a function of dimensionless residence time reported.

Our results identified two cases of practical interest. The first feature corresponds to the case where solutions to both no-washout and washout conditions are bistable. The second feature identifies the parameter region in which periodic solutions can occur when the yield coefficient is not constant. Both these features are often undesirable in practical applications and must be avoided.

Scaling of the model equations reveals that both the second-bifurcation parameters are functions of the influent concentration. Our results predict how the reactor behaviour varies as a function of influent concentration and identify the range of influent concentration where the reactor displays neither periodic nor bistable behaviour.
\end{abstract}


Keywords: Bioreactor; Chemostat; Contois growth kinetics; the noncompetitive substrate inhibition; Water treatment.

\section{Introduction}

The functioning of a bioreactor lies principally in understanding the growth rate of the biomass. Many models proposed in the literature, including those of Tessier (Tessier, 1936), Monod (Monod, 1949), Moser (Moser, 1958) and Contois (Contois, 1959), address the kinetics of the growth of the cell mass in the bioreactor and most of these models report expressions for specific growthrate of cell mass. However many industrial wastes contain chemical species such as ammonia, nitrates, phenols, thiocyanates, and volatile acids which are known to have inhibitory effects on the growth of the biomass. Hence a growth rate expression that includes the inhibitory effect of the substrate concentration on the growth of the biomass is often required to accurately model the treatment of industrial wastes.

Several empirical models that capture the features of substrate inhibition effects on microbial growth have been proposed in the literature [Michael \& Fikret (2002), page 179]. Andrews (1968) was the first to propose an equation to model substrate-inhibited microbial growth and his model provided a good fit to the experimental data and has been widely used particularly for wastewater treatment systems (Sokol, 1987; Grady \& Lim, 1999; Tang \& Fan, 1987; Rozich \& Gaudy Jr, 1985; Goudar et al., 2000; Tan et al., 1996). Andrew's equation was basically an extension of the Monod model (Monod, 1949) where an additional substrate inhibition term has been included $\left(S^{2} / K_{I}\right)$. However, as described in section (2), there are a number of industrial processes, in particular wastewater treatment, which use Contois kinetics (Contois, 1959) model for cell-growth rate since Contois model is more accurate than Monod kinetics. Thus, it is reasonable to assume that the Contois model can be improved by an inclusion of similar substrate inhibition term $\left(S^{2} / K_{I}\right)$.

Several workers also suggest that, the experimentally found oscillatory behaviour of microbial population can be explained when the yield coefficient is dependent on the substrate concentration (Dorofeev et al., 1992; Lee et al., 1976) and not when the yield coefficient is constant. A number of theoretical studies have examined the consequences of this assumption, these are discussed in section (2.2). Therefore, in order to examine the most general growth 
rate based on the Contois expression kinetics that could be expected to occur in practise, we extend the Contois expression subject to include both substrate inhibition and variable yield.

In this paper, we investigate the steady-state behaviour of a biological process in a generalized bioreactor model in which the specific growth-rate is given by a Contois expression (Contois, 1959) subject to substrate inhibition with a variable yield coefficient. Both the static and dynamic behaviours are investigated using this generalized growth-rate expression. We determine the stability of steady-state solutions, report asymptotic solutions in the limit of high residence times and discuss conditions where the bioreactor shows bistable (i.e. where the no washout solution and the washout solution are both stable at the same value of the residence time) and periodic behaviours. When the reactor is bistable, care must be taken to ensure that random perturbations do not move the system from the basin of attraction of the no-washout solution to the basin of attraction of the washout solution. Similarly periodic behaviour is not desirable in many applications. Thus we believe, the main contribution of this study is identifying the parametric space where such undesirable behaviours occur during static and dynamic operations of a bioreactor and offering recommendations on suitable parametric space where bioreactor could successfully operate in.

In this paper we use standard techniques from the theory of non-linear dynamical systems, including the singularity theory and Hopf bifurcation theory. For the reader who is unfamiliar with these techniques, we recommend a recently published book (Ajbar \& Alhumaizi, 2011) which uses these theories to analysis the dynamic and static behavior of many chemostat. This emphasizes the interpretation of mathematical result in terms of the underlining of chemical engineering problem.

\section{Literature review on Contois growth kinetics and model with variable substrate yield}

An overview of recent experimental work using the Contois growth model is provided in section (2.1). In section (2.2) we outline some of the investigations that have been carried out into systems with a variable yield. 
Table 1: Industrial wastewaters described by Contois model

\begin{tabular}{|c|c|c|}
\hline Substrate & Other Model & Reference \\
\hline Ice-cream wastewater & Monod kinetic & Hu et al. (2002) \\
\hline Textile wastewater & Monod kinetic & Işik \& Sponza (2005) \\
\hline $\begin{array}{l}\text { Synthetic domestic wastewater } \\
\text { (hydrolytic stage, two reaction) }\end{array}$ & - & Guerrero et al. (2009) \\
\hline $\begin{array}{l}\text { Penicillium } \quad \text { brevicom- } \\
\text { pactum(filamentous fungi)ask }\end{array}$ & $\begin{array}{l}\text { Verhulst and Exponential } \\
\text { models }\end{array}$ & Ardestani (2012) \\
\hline $\begin{array}{l}\text { Waste activated sludge [hydroly- } \\
\text { sis] }\end{array}$ & $\begin{array}{l}\text { First order reaction (Monod } \\
\text { kinetic) and Hill function }\end{array}$ & Ramirez et al. (2009) \\
\hline Caffeic Acid & Monod kinetic & Pinna et al. (2009) \\
\hline Palm oil mill effluent wastewater & $\begin{array}{l}\text { Monod and Chen and } \\
\text { Hashimoto }\end{array}$ & Abdurahman et al. (2011a) \\
\hline Palm oil mill effluent wastewater & $\begin{array}{l}\text { Monod and Chen and } \\
\text { Hashimoto }\end{array}$ & Abdurahman et al. (2011) \\
\hline Palm oil mill effluent wastewater & $\begin{array}{l}\text { Chen and Hashimoto's ki- } \\
\text { netic }\end{array}$ & Poh \& Chong (2010) \\
\hline Synthesised dairy wastewater & $\begin{array}{l}\text { Monod, Moser, and Chen } \\
\text { and Hashimoto }\end{array}$ & Emerald et al. (2012) \\
\hline Paper sludge & Monod kinetic & Zhang et al. (2009) \\
\hline $\begin{array}{l}\text { The decomposition processes of } \\
\text { mining influenced water, sulfate }\end{array}$ & The first order model & Hemsi et al. (2010) \\
\hline Pharmaceutical wastewater & Tessier and Monod kinetics & Sun et al. (2009) \\
\hline
\end{tabular}

\subsection{Processes described by the Contois model}

The Contois growth rate expression has been shown experimentally to be applicable to both anaerobic and aerobic degradation of industrial wastewaters. Table (1) summarizes wastewater treatment applications in which the Contois model provided a good fit to the experimental data. Table (2) summarizes investigations in which the Contois model was found to describe processes other than the treatment of industrial wastewaters. The second column of tables (1 and 2) list other kinetic models that are compared against the Contois kinetic models.

Gawande et al. (2010) presented the development of a generalized biochemical process model. They modeled the hydrolysis of particulate matter using Contois kinetics, described the microbiologically mediated reaction and simulated the anaerobic reduction of municipal solid waste. They observed that Contois kinetics gave predicted the reduction of solid concentrations very well. The Contois growth rate has been used as a default growth-rate model in simulations of the cleaning of wastewater by microorganisms (Czeczot et al., 2000), a further indication that this model is relevant and appropriate to be used to model industrial wastewater processes. The Contois model has also been receiving growing attention in ecology (Abrams \& Ginzburg, 2000; Cabrera \& Maria, 2011; Harmand \& Godon, 2007). 
Table 2: Other processes governed by the Contois model

\begin{tabular}{|l|l|l|}
\hline Application & Other Model & Reference \\
\hline $\begin{array}{l}\text { The lactate fermentation charac- } \\
\text { teristics[hydrolysis], the hydroly- } \\
\text { sis of particulate kitchen garbage }\end{array}$ & $\begin{array}{l}\text { First order reac- } \\
\text { tion (Monod kinetic) }\end{array}$ & Hidaka et al. (2010) \\
\hline $\begin{array}{l}\text { Rhizopus nigricans fungal on glu- } \\
\text { cose [Fungal growth] }\end{array}$ & - & Zhou et al. (2009) \\
\hline Inulinase production & 19 kinetic models & Mazutti et al. (2009) \\
\hline Extracellular enzyme & Monod kinetics & Hernalsteens \& Maugert (2010) \\
\hline Algae growth & - & Ilichev (2008) \\
\hline Composting & Monod kinetics & Bongochgetsakul \& Ishida (2008) \\
\hline $\begin{array}{l}\text { Penicillium chrysogenum grown } \\
\text { on glucose [Fungal growth] }\end{array}$ & - & Goudar \& Strevett (1998) \\
\hline
\end{tabular}

\subsection{Models with a variable yield coefficient}

In many models of bioreactors, it is assumed that the yield coefficient is a constant during the course of the reaction. However, some experimental evidence has shown that the yield coefficient can be function of substrate concentration. In this section, we provide a brief overview of models using a variable substrate yield by extending the literature review appearing in (Nelson \& Sidhu, 2009).

Ajbar (2010) analysed a continuous bioreactor using a general dependence for the growth rate and a variable yield coefficient upon the substrate concentration. The author consider a non-ideal bioreactor in which the bioreactor was divided into two regions, a well-mixed region and an unreacted region. This study showed that the variability of the substrate yield is a necessary condition for the existence of periodic behavior and it extends earlier finding of Crooke et al. (1980), that showed the periodic solutions cannot occur for systems with a constant yield, from an ideal reactor to a non-ideal reactor.

Zhu (2007) investigated a bioreactor in which the growth rate was assumed to depend only on the substrate concentration and claimed that limit cycles can occur in a chemostat with two competitors for a single nutrient when there is a constant substrate yield. However, Sari (2010a) re-investigated the reactor model of Zhu (2007) and showed that there was an error in the computation of the eigenvalues in $(\mathrm{Zhu}, 2007)$ and that consequently the result in (Zhu, 2007 ) is false. In this study the growth rate was assumed to depend only on the substrate concentration.

A number of authors have studied the behavior of a chemostat with two microorganisms 
and a single substrate with variable yields (Song \& Li, 1999; Huang \& Zhu, 2005, 2009). Song \& Li (1999) assumed that the growth rate follows Monod kinetics with a variable substrate yield. Huang \& Zhu (2005) extended the study (Song \& Li, 1999) by considering to quadratic substrate yield. Huang \& Zhu (2009) furthermore generalised the two linear yield functions to nth and mth order-polynomials and replaced the standard Monod specific growth rate by a general non deceasing function. Sari (2010b) studied a chemostat model in which n species compete for a single growth-limiting substrate by extending the results of (Wolkowicz \& Lu, 1992) to the case when yields are variable. The growth rate includes both monotone and non-monotone response functions (which also includes Monod kinetics).

A number of authors have investigated the behavior of reactor in which the specific growth rate follows the Contois model with a linear yield coefficient (Nelson \& Sidhu 2007; Ajbar 2012; Ajbar et al. 2011; Alqahtani et al. 2012; Nelson et al. 2008a). Nelson \& Sidhu (2007); Nelson et al. (2008a) analysed a well-mixed continuously stirred reactor and membrane reactor respectively. They found the parameter region in which oscillations may be generated in the reactor and compared the performance of a single reactor against that of a cascade of two reactors. Ajbar et al. (2011) extended the study (Nelson \& Sidhu, 2007) to take into account oxygen transfer limitations. Alqahtani et al. (2012) also extended the work of (Nelson \& Sidhu, 2007) by including the death rate of the microorganisms. Ajbar (2012) considered a chemostat model in which two microbial populations compete for the same substrate with a two different variable yield coefficient. In this study, the specific growth rates of both species are assumed to follow the Contois model. The analysis of this model shows that the assumption of non-constant yield may lead to complex behavior such as chaotic behavior.

\section{Model equations}

A system of nonlinear ordinary differential equations model the biodegradation of organic substances in wastewaters in which cell mass (X) grows through consumption of a substrate species (S). The specific growth rate, equation (3), is given by a Contois expression subject to substrate inhibition. The yield coefficient is a function of the substrate, equation (5). The dependent variables are the concentration of organic substrate $(\mathrm{S})$ and the concentration of the degrader $(\mathrm{X})$, the independent variable is time $(\mathrm{t})$. 
The dimensional and dimensionless forms of our model are stated in sections (3.1) and (3.2), respectively.

\subsection{The dimensional model}

The governing equations for a single reactor arise from a mass balance on the substrate and biomass and are given by the following equations

$$
\begin{aligned}
V \frac{d S}{d t} & =F\left(S_{0}-S\right)-V X \frac{\mu(S, X)}{Y(S)}, \\
V \frac{d X}{d t} & =F\left(X_{0}-\beta X\right)+V X \mu(S, X) .
\end{aligned}
$$

The Contois expression with substrate inhibition is given by

$$
\mu=\mu_{m}\left(\frac{S}{K_{s} X+S+\frac{S^{2}}{K_{I}}}\right) .
$$

It can be noted that in the limit $K_{I} \rightarrow \infty$ we obtain the standard Contois model.

The residence time is,

$$
\tau=\frac{V}{F}
$$

The yield coefficient is

$$
Y(S)=\alpha+\gamma S, \quad(\alpha, \gamma>0)
$$

Note that the standard constant yield coefficient corresponds to the case $\gamma=0$. The terms that appear in equations (1) and (2) are defined in appendix A.

We consider a general reactor model where the choice of value for parameter $\beta$ determines the configuration of the reactor. When $\beta=1$ the bioreactor is a continuous flow reactor. When $\beta=0$ it is an idealized membrane reactor, in which all of the microorganisms is forced to remain in the reactor vessel. The choice $0<\beta<1$ gives a non-idealised membrane reactor which is equivalent to a reactor with recycle because the parameter $\beta$ is related to the recycle parameter as $\beta=1-R^{*}$ (Nelson et al. 2008b) where $R^{*}$ is the recycle parameter. In this case some of 
the microorganisms leave the reactor vessel and some of them stay at the reactor vessel. For a non-idealised membrane reactor to be operationally effective we require $0<\beta \ll 1$. Since, the death of microorganisms is not included in the model thus $\beta=0$ cannot be made.

The model (1 and 2) does not include biomass decay and has previously been considered for the case of a yield coefficient $(\gamma=0$ and $e=0)$ in (Nelson et al. 2008b). In Nelson et al. (2008a); Nelson \& Sidhu (2007) a variable yield was used but microbial inhibition was not included in model $\left(\gamma^{*}>0, K_{I}=\infty\right)$. Thus this study both extends previous work and provides a useful first step towards studying a more complicated model that includes microbial inhibition.

\subsection{The dimensionless model}

The system of differential equations $(1,2)$ can be written in dimensionless form by introducing dimensionless variables for substrate concentration $\left[S^{*}=S / S_{0}\right]$, the microorganism concentration $\left[X^{*}=K_{s} X / S_{0}\right]$, and time $\left[t^{*}=\mu_{m} t\right]$.

$$
\begin{aligned}
\frac{d S^{*}}{d t^{*}} & =\frac{1}{\tau^{*}}\left(1-S^{*}\right)-\frac{S^{*} X^{*}}{\left(\alpha^{*}+\gamma^{*} S\right)\left(S^{*}+X^{*}+e S^{* 2}\right)}, \\
\frac{d X^{*}}{d t^{*}} & =\frac{X_{0}^{*}-\beta X^{*}}{\tau^{*}}+\frac{S^{*} X^{*}}{\left(S^{*}+X^{*}+e S^{* 2}\right)} .
\end{aligned}
$$

In these equations $(6,7), X_{0}^{*}$ is the dimensionless microorganism concentration in the feed $\left(X_{0}^{*}=\right.$ $\left.X_{0} / \alpha K_{s}\right), \alpha^{*}$ is the dimensionless yield coefficient $\left(\alpha^{*}=K_{s} \alpha\right), \tau^{*}$ is dimensionless resident time $\left(\tau^{*}=V \mu_{m} / F\right), e$ is the dimensionless inhibition constant $\left(e=S_{0} / K_{I}\right)$ and $\gamma^{*}$ is the dimensionless linear yield coefficient $\left(\gamma^{*}=\gamma K_{s} S_{0}\right)$.

This model is investigated with the assumptions that the concentration of microorganisms flowing into the reactor is zero $\left(X_{0}^{*}=X_{0}=0\right)$ and the concentration of substrate entering into the reactor is positive $\left(S_{0}>0\right)$.

We take the dimensionless residence time as the primary bifurcation parameter. The dimensionless constant in the yield coefficient $\left(\gamma^{*}\right)$ and the dimensionless inhibitions term $(e)$ are the secondary bifurcation parameters. The values for $\alpha^{*}$ are determined by the choice of microbial system and is therefore not a "tunable" parameter. Note that the scaled kinetics parameters $\gamma^{*}$ and e, which control the nonlinear terms in the model equation, are functions 
of the influent concentration $\left(S_{0}\right)$. Thus varying the influent concentration, changes the significance of the nonlinear terms. Since there is a one to one relationship between our dimensionless variables and their dimensional counterparts, often we refer to either with the same name while discussing results.

\section{Results}

In section (4.1) the steady state solutions branches are found. In section (4.2), we study the stability of the steady state solutions. In section (4.3), we discuss the occurrence of degenerate Hopf bifurcations along the no-washout state and the steady state diagrams. In section (4.4) asymptotic solutions for large residence times are stated.

The XPP software package (Ermentrout, 2002) was used to obtain the steady-state diagrams. The standard representation is used in these: solid lines are stable steady states; dotted lines are unstable steady states; filled-in squares are Hopf bifurcation points; open circles are unstable periodic orbits; filled-in circles are stable periodic solutions. We use the symbol open triangles to denote a limit point, filled-in triangles for other static bifurcation points and open squares for a double-zero eigenvalue. For a periodic orbit the average that is used is the integral over the period of the solution.

\subsection{Steady State Solutions Branches}

The steady state solutions of the system of equations $(6,7)$ are given by, The washout branch,

$$
\left(S^{*}, X^{*}\right)=(1,0)
$$

The no-washout branch,

$$
\begin{aligned}
\left(S^{*}, X^{*}\right) & =\left(\widehat{S_{i}^{*}}, \frac{\left(\alpha^{*}+\gamma^{*} \widehat{S_{i}^{*}}\right)\left(1-\widehat{S_{i}^{*}}\right)}{\beta}\right), \\
\widehat{S_{1}^{*}} & =\frac{-b}{2 a}-\frac{\sqrt{b^{2}-4 a c}}{2 a} \quad \text { and } \quad \widehat{S_{2}^{*}}=\frac{-b}{2 a}+\frac{\sqrt{b^{2}-4 a c}}{2 a} .
\end{aligned}
$$


The values $\widehat{S_{i}^{*}}(\mathrm{i}=1,2)$ are the roots of the quadratic equation

$$
F=a{\widehat{S^{*}}}^{2}+b \widehat{S^{*}}+c=0
$$

The coefficients a, b, and c are defined by

$$
a=e \beta-\gamma^{*}, \quad b=-\left(\tau^{*}+\alpha^{*}-\beta\right)+\gamma^{*}, \quad c=\alpha^{*} .
$$

We have the following restriction on parameter values: $e>0, \gamma^{*}>0,0 \leq \beta \leq 1, \tau^{*}>0$. Equation (10) defines the substrate concentration $\left(S^{*}\right)$ as an implicit function of the residence time $\left(\tau^{*}\right)$. We are interested for the positive value of substrate concentration $\left(S^{*}\right)$. Since, equation (10) is quadratic in $S^{*}$, it has one, or more, positive solutions. Note that the coefficient c strictly positive consequently $S^{*}=0$ is never to be a solution of equation (10). In order to analysis the equation (10), we consider three possible cases: $a<0, a=0$ and $a>0$.

Case 1: $a<0$. This case happens when

$$
\gamma^{*}>e \beta
$$

or in the original variables when

$$
\left(\beta-\gamma K_{s} K_{I}\right)<0
$$

Inequality (11) is always satisfied for a perfect membrane reactor $(\beta=0)$. When $a<0$, equation (10) has two real solutions with $\widehat{S_{2}^{*}}<0<\widehat{S_{1}^{*}}$. Restricting our attention to the physically meaningful situation we see that equation (10) has one positive real solution, $\widehat{S_{1}^{*}}$ and only physically meaningful solution when $\tau^{*}>\tau_{\mathrm{BR}}^{*}$.

Case 2: $a=0$. This case happens when

$$
\gamma^{*}=e \beta,
$$

or in the original variables when

$$
\left(\beta-\gamma K_{s} K_{I}\right)=0
$$

Equality (13) is never satisfied for a perfect membrane reactor $(\beta=0)$. When $a=0$, equa- 
tion (10) has one real solution given by

$$
\widehat{S^{*}}=\frac{1}{\frac{\tau^{*}-\beta(e+1)}{\alpha^{*}}+1} .
$$

which is only physically meaningful solution when $\tau^{*}>\beta(1+e)$.

Case 3: $a>0$. This case happens when

$$
\gamma^{*}<e \beta,
$$

or in the original variables when

$$
\left(\beta-\gamma K_{s} K_{I}\right)>0
$$

Inequality (16) is never satisfied for a perfect membrane reactor $(\beta=0)$. In the appendix $(A)$ we show that the solutions of the equation (10) are only of interest when

$$
\tau^{*} \geq \tau_{1}^{*}=\left(\beta+\gamma^{*}-\alpha^{*}\right)+2 \sqrt{\alpha^{*}\left(e \beta-\gamma^{*}\right)}
$$

The solutions of the quadratic equation (10) are both positive (with $S_{2}^{*} \geq S_{1}^{*}$ ) when the condition (18) holds, but they may not correspond to physically meaningful solutions.

It is instructive to investigate how the effluent concentration on the non-washout state changes as the residence time varied. Differentiating equation (10) we obtain

$$
\frac{d S^{*}}{d \tau^{*}}=\frac{S^{*}}{2 a S^{*}+b}= \pm \frac{S^{*}}{\sqrt{b^{2}-4 a c}}
$$

In equation (19) the choice of the sign $+/-$ corresponds to the choice of $S_{2}^{*}$ or $S_{1}^{*}$ respectively.

For physically meaningful solutions, equation (19) is negative along the solution branch $S_{1}^{*}$ and positive along the solution branch $S_{2}^{*}$. Thus, the solution branch $S_{1}^{*}$ is an decreasing function of the residence time while the solution branch $S_{2}^{*}$ is an increasing function of the residence time.

In the following we apply singularity theory to the quadratic equation (10). The only possible bifurcation point that can occur in the steady state solution is the limit point. We establish later that for certain parameter values where the limit point can occur, reactor becomes bistable which is undesirable in many applications. The limit point occurs when (Ajbar \& 
Alhumaizi, 2011),

$$
F=F_{\widehat{S^{*}}}=0
$$

The non-denegencay conditions are (Ajbar \& Alhumaizi, 2011):

$$
F_{\widehat{S^{*}} \widehat{S^{*}}} \neq 0 \quad \text { and } \quad F_{\tau^{*}} \neq 0
$$

The condition $F=F_{\widehat{S^{*}}}=0$ implies that

$$
\left(S^{*}, \tau^{*}\right)=\left(\sqrt{\frac{\alpha^{*}}{e \beta-\gamma^{*}}}, \tau_{1}^{*}\right)
$$

This condition is satisfied only if $e \beta-\gamma^{*}>0$. Thus there is no limit point bifurcation when $a \leq 0$ (cases 1,2). The non-denegencay conditions are satisfied at equation (22) as,

$$
\begin{aligned}
F_{S^{*} S^{*}} & =2 a \neq 0 \quad \text { provided } \quad e \beta-\gamma^{*} \neq 0, \\
F_{\tau^{*}} & =-S^{*} \neq 0 .
\end{aligned}
$$

We conclude that the necessary condition for the existence of the limit point in equation (22) is $a>0$ (Case 3). Henceforth we denote the value of the substrate concentration at the limit point by $S_{\mathrm{LP}}^{*}$. Note that the limit point occurs on the washout line $\left(S_{\mathrm{LP}}^{*}=1\right)$ and leads to the simple condition,

$$
e=e_{\mathrm{cr}, 1}=\frac{\alpha^{*}+\gamma^{*}}{\beta}
$$

This condition defines the transition between two types of static behavior. If the substrate inhibition parameter $(\mathrm{e})$ is below the critical value $\left(e<e_{\mathrm{cr}, 1}\right)$ then the limit point is physically unrealistic whereas, if the substrate inhibition parameter $(\mathrm{e})$ is above the critical value $\left(e>e_{\mathrm{cr}, 1}\right)$ then the limit point is physically realistic. In practical applications it is more useful to turn the dimensionless critical value, $e_{\mathrm{cr}, 1}$ to the critical value of the influent concentration. Returning 
to dimensional values, we find that,

$$
S_{0, \mathrm{cr}}=\frac{\alpha K_{s} K_{I}}{\beta-\gamma K_{s} K_{I}}
$$

If the influent concentration is below this critical value $\left(S_{0}<S_{0, \mathrm{cr}}\right)$ then the limit point, if it is exists, is physically unrealistic, we are to the left of line B in figure 2(a). On other hand, if the influent concentration is above this critical value $\left(S_{0}>S_{0, \mathrm{cr}}\right)$ then the limit point is physically realistic, we are to the right of line $\mathrm{B}$ in figure $2(\mathrm{a})$, consequently the bistability occurs in the steady state diagram. Once should be noted from inequality (17) that the existence of the limit point requires the following condition, $\beta-\gamma K_{s} K_{I}>0$. If inequality (17) does not hold then there is no bistabile solution.

A transcritical bifurcation in which the solution of equation (10) intersects the washout line $\left(S^{*}=1\right)$ is given by,

$$
\tau^{*}=\tau_{B R}^{*}=\beta(1+e)
$$

A pitchfork bifurcation on other hand occur when $\tau_{B R}^{*}=\tau_{1}^{*}$.

It is useful to draw the location of the possible steady state and bifurcation points that may occur in the model. Figure 1 shows the steady state solutions, the realistic and unrealistic limit point in the parameter space $\left(\gamma^{*}, e\right)$. It can be seen from the figure 1 that the existence of the realistic limit point and the bistable solution for the cases $(1$ and 2$)(a \leq 0)$ are impossible. Simple analysis of the case $3(a>0)$ reveals three different possible cases depending on the value of the limit point.

Case 3 A. $S_{\mathrm{LP}}^{*}>1$.

This case corresponds to the condition $\frac{\gamma^{*}}{\beta}<e<\frac{\alpha^{*}+\gamma^{*}}{\beta}$. For values of the residence time for which either $S_{2}^{*}>S_{\mathrm{LP}}^{*}>1$ or $S_{\mathrm{LP}}^{*}>S_{1}^{*}>1$ then, from equation (9), the corresponding value for the microorganism concentration is negative. Thus, the solution branch $S_{2}^{*}$ is not physically realistic. The range of the residence time at which $S_{\mathrm{LP}}^{*}>S_{1}^{*}>1$ is $\tau_{1}^{*}<\tau^{*}<\tau_{\mathrm{BR}}^{*}$. Over this parameter range, the solution branch $S_{1}^{*}$ is also not physically realistic, but it is a physically realistic when $\tau^{*}>\tau_{\mathrm{BR}}^{*}$ because here $S_{1}^{*}<1$ and hence $X_{1}^{*}>0$. Note that a transcritical bifurcation and a physically unrealistic limit point bifurcation occur at $\tau^{*}=\tau_{\mathrm{BR}}^{*}$ and $\tau^{*}=\tau_{1}^{*}$ 
respectively. Thus the existence of the bistable solution is impossible.

Case 3 B. $S_{\mathrm{LP}}^{*}=1$.

This case corresponds to the condition $e=\frac{\alpha^{*}+\gamma^{*}}{\beta}$. The solution branch $S_{2}^{*}$ is not physically realistic because $S_{2}^{*}>S_{\mathrm{LP}}^{*}>1$. However, the solution branch $S_{1}^{*}$ is physically realistic for $\tau^{*}>\tau_{\mathrm{BR}}^{*}$. One notice that transcritical bifurcation is equal to limit point bifurcation consequently a pitchfork singularity occur at $\tau^{*}=\tau_{\mathrm{BR}}^{*}=\tau_{1}^{*}$. Thus the existence of the bistable solution is impossible.

Case 3 C. $S_{\mathrm{LP}}^{*}<1$.

This case corresponds to the condition $e>\frac{\alpha^{*}+\gamma^{*}}{\beta}$. The solution branch $S_{2}^{*}$ is only physically realistic for the residence time $\tau^{*}$ when $\tau_{1}^{*}<\tau^{*}<\tau_{\mathrm{BR}}^{*}$. It is not physically realistic for $\tau^{*}>\tau_{\mathrm{BR}}^{*}$. The solution branch $S_{1}^{*}$ is a physically realistic when $\tau^{*}>\tau_{1}^{*}$. Thus equation (10) has two physically realistic solutions if $\tau_{1}^{*}<\tau^{*}<\tau_{\mathrm{BR}}^{*}$ and one if $\tau^{*}>\tau_{\mathrm{BR}}^{*}$. Note that a transcritical bifurcation and physically realistic limit point bifurcation occur at $\tau^{*}=\tau_{\mathrm{BR}}^{*}$ and $\tau^{*}=\tau_{1}^{*}$ respectively. Thus the bistable solution corresponds to the range of the residence time $\tau_{1}^{*}<\tau^{*}<\tau_{\mathrm{BR}}^{*}$

In this case there is a range of the residence time $\left(\tau_{1}^{*}<\tau^{*}<\tau_{\mathrm{BR}}^{*}\right)$ in which the two solutions, the washout and the no washout, are stable. This implies that a small random perturbation can cause the reactor operating at the no-washout branch to move and operate and the washout branch, i.e. the process failure occurs in the reactor. In practices it may be desirable to avoid the bistable region. This always can be achieved by operating the reactor at large residence time $\left(\tau^{*}>\tau_{\mathrm{BR}}^{*}\right)$.

\subsection{Stability of the steady state solutions}

The study of the stability of the solution is important because only the stable solution maybe observed experimentally. The stability of the steady state solutions for the system of equations $(6,7)$ is determined by the eigenvalues of the Jacobian matrix evaluated at the steady state solutions. The Jacobian matrix is given by

$$
\mathrm{J}\left(S^{*}, X^{*}\right)=\left[\begin{array}{cc}
-\frac{1}{\tau^{*}}+\frac{X^{*}\left(2 e \gamma^{*} S^{*^{3}}+S^{*^{2}}\left(\gamma^{*}+\alpha^{*} e\right)-\alpha^{*} X^{*}\right)}{\left(\alpha^{*}+\gamma^{*} S^{*}\right)^{2}\left(X^{*}+S^{*}+e S^{*^{2}}\right)^{2}} & -\frac{\left(1+e S^{*}\right) S^{*^{2}}}{\left(\alpha^{*}+\gamma S^{*}\right)\left(X^{*}+S^{*}+e S^{* 2}\right)^{2}} \\
-\frac{X^{*}\left(-X^{*}+e S^{*}\right)}{\left(X^{*}+S^{*}+e S^{2}\right)^{2}} & -\frac{\beta}{\tau^{*}}+\frac{S^{* 2}\left(1+e S^{*}\right)}{\left(X^{*}+S^{*}+e S^{* 2}\right)^{2}}
\end{array}\right]
$$




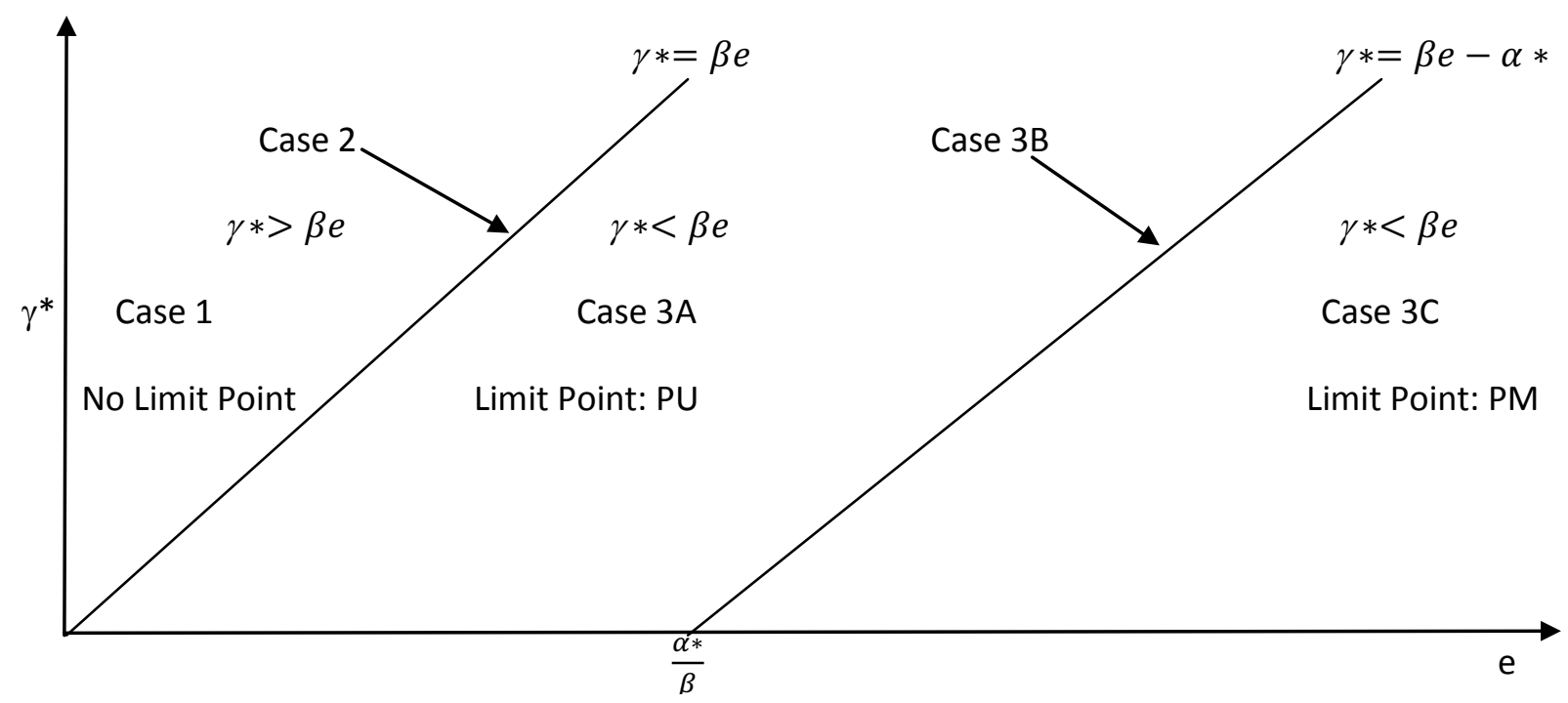

Figure 1: Schematic diagram showing the location of the different type of steady state diagrams in the parameter space $\left(\gamma^{*}, e\right)$. PU=physically unmeaningful, $\mathrm{PM}=$ physically meaningful.

\subsubsection{Stability along the washout branch}

Under washout conditions the effluent concentration is equal to the inflow concentration. Thus this state of operation must be avoided. Evaluating the Jacobian matrix (26) at the washout steady state solution gives,

$$
J(1,0)=\left[\begin{array}{cc}
-\frac{1}{\tau^{*}} & -\frac{1}{\left(\alpha^{*}+\gamma^{*}\right)(1+e)} \\
0 & -\frac{\beta}{\tau^{*}}+\frac{1}{1+e}
\end{array}\right]
$$

The eigenvalue of this matrix are given by,

$$
\lambda_{1}=-\frac{1}{\tau^{*}}<0, \quad \lambda_{2}=\frac{-\beta(1+e)+\tau^{*}}{\tau^{*}(1+e)} .
$$

It follows that the washout branch is a stable provided that

$$
\tau^{*}<\beta(1+e)=\tau_{\mathrm{BR}}^{*}
$$

Equation (28) established a condition of the residence time at which the process fails occur in the reactor. When the inequality (28) is hold then due to the process failure the rate of microorganisms that leaving the reactor is greater than their maximum growth rate. Equation (28) also shows that a perfect membrane cannot have washout, a good membrane could only have washout at a very small residence time as $\tau_{\mathrm{BR}}^{*}=0$. 


\subsubsection{Stability along the no-washout branch}

In this section, we investigate the stability of the no-washout state by evaluating the Jacobian matrix at the no-washout state. We find that

$$
\mathrm{J}\left(S^{*}, X^{*}\right)=\left[\begin{array}{ll}
J_{11} & J_{12} \\
J_{21} & J_{22}
\end{array}\right]
$$

where

$$
\begin{aligned}
& J_{11}=-\frac{1}{\tau^{*}}+\frac{X^{*}\left(2 e \gamma^{*} S^{*^{3}}+S^{*^{2}}\left(\gamma^{*}+\alpha^{*} e\right)-\alpha^{*} X^{*}\right)}{\left(\alpha^{*}+\gamma^{*} S^{*}\right)^{2}\left(X^{*}+S^{*}+e S^{*^{2}}\right)^{2}}, \\
& J_{12}=-\frac{\left(1+e S^{*}\right) S^{*^{2}}}{\left(\alpha^{*}+\gamma S^{*}\right)\left(X^{*}+S^{*}+e S^{*^{2}}\right)^{2}}, \\
& J_{21}=-\frac{X^{*}\left(-X^{*}+e S^{*^{2}}\right)}{\left(X^{*}+S^{*}+e S^{*^{2}}\right)^{2}} . \\
& J_{22}=-\frac{X^{*} S^{*}}{\left(X^{*}+S^{*}+e S^{*^{2}}\right)^{2}}
\end{aligned}
$$

To obtain the afore-given expression, we have used the fact that along the no-washout branch,

$$
\frac{-\beta}{\tau^{*}}=-\frac{S^{*}}{\left(X^{*}+S^{*}+e S^{* 2}\right)} .
$$

The case $2(\mathrm{a}=0)$ is considered in the appendix $(\mathrm{C})$. We evaluate the determinant of matrix $(J)$ for the case when $a \neq 0$ (Cases 1 and 3). After some algebra we find,

$$
\begin{aligned}
\operatorname{det}(\mathrm{J})\left(\widehat{S_{1}^{*}}, \widehat{X_{1}^{*}}\right) & =-\frac{2 \beta\left(e \beta-\gamma^{*}\right)\left[(\Delta+b \sqrt{\Delta})\left(1-S_{1}^{*}\right)\right]}{\tau^{*^{3}}(b+\sqrt{\Delta})^{2}}, \\
\operatorname{det}(\mathrm{J})\left(\widehat{S_{2}^{*}}, \widehat{X_{2}^{*}}\right) & =-\frac{2 \beta\left(e \beta-\gamma^{*}\right)\left[(\Delta-b \sqrt{\Delta})\left(1+S_{2}^{*}\right)\right]}{\tau^{*^{3}}(-b+\sqrt{\Delta})^{2}} .
\end{aligned}
$$

The expression $(\Delta)$ is defined by equation (50) and it is always non-negative $(\Delta \geq 0)$. For case $(1)(a<0)$, the solution, $\left(\widehat{S_{2}^{*}}, \widehat{X_{2}^{*}}\right)$, is physically unrealistic so we ignore it. However, along the physically meaningful solution, $\left(\widehat{S_{1}^{*}}, \widehat{X_{1}^{*}}\right)$, the following expression, $\Delta+b \sqrt{\Delta}$, is positive. It follows that the determinant of matrix $(J)$ along the physically meaningful solution, $\left(\widehat{S_{1}^{*}}, \widehat{X_{1}^{*}}\right)$ 
is positive.

For case $3(a>0)$, the both solutions are considered and a necessary condition for either of these solutions to be physically realistic is $b<0$ (See appendix B). Thus, along the physically meaningful solution, $\left(\widehat{S_{2}^{*}}, \widehat{X_{2}^{*}}\right)$, the following expression, $\Delta-b \sqrt{\Delta}$, is positive. It follows that the determinant of matrix $(J)$ along the physically meaningful solution, $\left(\widehat{S_{2}^{*}}, \widehat{X_{2}^{*}}\right)$ is negative. However, the following expression, $\Delta+b \sqrt{\Delta}$ is negative for $b<0$ (See appendix D). It follows that the determinant of matrix $(J)$ along the physically meaningful solution, $\left(\widehat{S_{1}^{*}}, \widehat{X_{1}^{*}}\right)$ is positive.

Therefore we conclude that for physically meaningful solution, the determinant of the matrix $(J)$ is positive along the branch solution $\widehat{S_{1}^{*}}$ and negative along the branch solution $\widehat{S_{2}^{*}}$ except when $\Delta=0$. Note that Hopf bifuraction can not occur along the branch solution $\widehat{S_{2}^{*}}$ (because $\operatorname{det}(J)<0)$ and the determinant of the matrix $(J)$ is equal to zero, when $\tau^{*}=\tau_{1}^{*}$.

\subsection{Degenerate Hopf bifurcation Analysis}

The conditions of a Hopf bifurcation are that (Gray \& Roberts, 1988),

$$
J_{11} J_{22}-J_{21} J_{12}>0, \quad \psi=J_{11}+J_{22}=0,
$$

The conditions for a double-zero eigenvalue degeneracy (DZE) to occur are given by (Gray \& Roberts, 1988),

$$
J_{11} J_{22}-J_{12} J_{21}=0, \quad \psi=J_{11}+J_{22}=0 .
$$

After some algebra the double-zero eigenvalue degeneracy is found to satisfy the equation,

$$
X^{*} S^{*^{2}}\left(e^{2} S^{*^{3}}+e S^{*^{2}}+X^{*}\left(\gamma^{*}-e\right) S^{*}+X^{*}\left(\alpha^{*}-1\right)\right)=0
$$

Note that $S^{*}=0$ does not correspond to a steady state solution of the model. The case $X^{*}=0$ correspond to the washout solution. We showed in section (4.2.1) that the washout solution always has a negative eigenvalue and consequently not exhibit the double zero eigenvalue 
degeneracy. Thus the double zero eigenvalue degeneracy must satisfy the equation,

$$
e^{2} S^{*^{3}}+e{S^{*}}^{2}+X^{*}\left(\gamma^{*}-e\right) S^{*}+X^{*}\left(\alpha^{*}-1\right)=0
$$

At a double-Hopf bifurcation, two Hopf points annihilate each other in an unfolding diagram (This is also known as $\mathrm{H} 2_{1}$ degeneracy). The conditions for the double-Hopf bifurcation to occur are given by (Gray \& Roberts, 1988),

$$
\operatorname{det}(\mathrm{J})=J_{11} J_{22}-J_{21} J_{12}>0, \quad \psi=J_{11}+J_{22}=0, \quad \frac{d \psi}{d \tau^{*}}=0
$$

Since it is not simple to analyse the conditions (33, 36 and 37) analytically, they have been solved numerically.

\subsubsection{Results: steady-state diagram}

Using a y-axis log scale in Figure (2), important features are displayed in the secondary bifurcation plane, i.e. $\quad \gamma^{*}, e$-plane. These features include the loci of the double-zero eigenvalue degeneracy (curve A), the line that is defined by equation (23) (line B) and two disjoint curves for the double-Hopf points (curve C). Each one of these features and the 6 regions bounded by line $\mathrm{B}$ and curves $\mathrm{A}$ and $\mathrm{C}$ will be discussed below. Note these regions are labeled from a to $\mathrm{g}$ and the steady state diagram in region (g) is identical to that in region (a).

The steady state diagram shows Hopf bifurcation points only in regions (b, c, d) and none in regions $(\mathrm{a}, \mathrm{g}, \mathrm{f})$. Using various parameter values for $e$ and $\gamma^{*}$, a more detailed characteristic or behavioral profile of the steady state diagram for each of the 6 regions, are presented in figure (3).

Above line (B) there may be a limit point, but it is not physically realistic. Conversely, below line (B), the limit point is realistic and the consequently the reactor exhibits bistability for some values of the residence time. This suggests that bistable condition of the reactor can be avoided by either using sufficiently low substrate inhibition $(e)$ or high substrate yield $\left(\gamma^{*}\right)$ parameters values. These parameters $\left(e, \gamma^{*}\right)$ turnout to be a function of the influent concentration $\left(S_{0}\right)$ and consequently the bistability can only be avoided if the influent concentration is sufficiently small. Thus, we conclude that the influent concentration must be controlled to ensure that the 
the reactor does not exhibit bistable behavior.

Note that the lower curve $\mathrm{C}$ in figure $(2(\mathrm{~b}))$ does not intersect with curve $\mathrm{A}$ and the end point of the lower curve $\mathrm{C}$ is obtained by,

$$
\left(e_{\mathrm{cr}, 2}, \gamma_{\mathrm{cr}, 2}^{*}\right) \approx(0.30364,0.01711)
$$

If the value of the substrate inhibition parameter (e) is higher than the critical value $\left(e_{\mathrm{cr}, 2}\right)$ then there is only one $\mathrm{H} 2{ }_{1}$ degeneracy point. For example, when $\alpha^{*}=0.01$ and $e=4$ the only solution satisfying the $\mathrm{H} 2{ }_{1}$ degeneracy condition (36) is

$$
\left(\gamma^{*}, \tau^{*}\right)=(14.88647,16.10751)
$$

Hence, the natural oscillations cannot occur on the steady state diagram when $\gamma^{*}>14.88647$ (region a only) but they may occur in other regions when $\gamma^{*}<14.88647$.

If the value of the substrate inhibition (e) is less than the critical value $\left(e_{\mathrm{cr}, 2}\right)$ then there are two $\mathrm{H} 2{ }_{1}$ degeneracy points. For example, in figure (2), when $\alpha^{*}=0.01$ and $e=0.1$ the $\mathrm{H} 2_{1}$ points are

$$
\left(\gamma^{*}, \tau^{*}\right)=(10.84049,12.05051), \quad\left(\gamma^{*}, \tau^{*}\right)=(0.020926,1.08110)
$$

Therefore, natural oscillations cannot occur in the regions $\left(0.02092>\gamma^{*}\right.$ and $\left.\gamma^{*}>10.84049\right)$. They occur in the region $\left(0.020926<\gamma^{*}<10.84049\right)$.

An example of region (a) is presented in figure 3(a). The region (a) is above the curves A,C and line B; thus neither a physically realistic limit point nor Hopf bifurcation points exist along the no washout solution branch.

On entering the region (b) from region (a), the $\mathrm{H} 2_{1}$ degeneracy curve is crossed consequently two Hopf bifurcations appear on the steady state diagram. Between the two Hopf points, the no washout solution is unstable. An example of this case is shown in figure 3(b).

Moving from region (b) to region (c), by crossing line (B), in figure (2(b)) the limit point bifurcation becomes physically realistic. The physically meaningful steady state diagram thus contains two Hopf bifurcations and a limit point. The upper branch of the no-washout solution is unstable while the lower branch is unstable between the two Hopf bifurcation points. An 
example of this case is shown in figure 3(c).

In figures 3(b) and 3(c), the periodic solution is stable. Between the two Hopf points, the average of the stable periodic solution is higher than the unstable steady-state solution. For this small region of operating space, the reactor operates at the stable periodic solution which increase the average effluent concentration leaving the reactor. We therefore conclude that in figure 3(b) and 3(c), the Hopf bifurcations have decreased the reactor performance.

When the parameter value moves into region (d) from region (c) by crossing the DZE locus (Curve A), one Hopf point is destroyed at a double-zero eigenvalue bifurcation and only one Hopf point remains as shown in figure $3(\mathrm{~d})$. The lower branch is unstable between the limit point and the Hopf bifurcation point. Furthermore the periodic solution is unstable and terminated at a homoclinic bifurcation.

Moving from region (c) into region (f), the remaining Hopf bifurcation point is destroyed and the steady state diagram contains a physically realistic limit point as shown in figure 3(e). Finally, moving from region (f) into region (g), we cross curve (B) and the limit point ceases to be physically realistic. The steady-state diagram in this case is identical to that in region (a), and is therefore not shown.

\subsubsection{Unfolding diagram for Hopf bifurcation}

Figure 4(a) is an unfolding diagram for the Hopf bifurcation point in Figure 3(c). It shows the value of the residence time at which a Hopf bifurcation point occurs as a function of $\gamma^{*}$. There are two Hopf bifurcation points in the region $\left(0.020926<\gamma^{*}<10.84049\right)$. The end points correspond to the $\mathrm{H} 2{ }_{1}$ degeneracy given by equation (40). In the region between the $\mathrm{H} 2_{1}$ degeneracies, periodic oscillations occur. For either large or small values of $\gamma^{*}$, including the constant yield case $\left(\gamma^{*}=0\right)$, there is no periodic behaviour. This example corresponds to the case $e<e_{\mathrm{cr}}$. (See section (4.3.1))

Figure 4(b) is an unfolding diagram for the Hopf bifurcation points in Figure 3(d) for the case $e>e_{\mathrm{cr}}$. There is only one Hopf bifurcation point in the region $0.01310<\gamma^{*}<3.89568$ and two Hopf bifurcation points in the range $3.89568<\gamma^{*}<14.88647$. A H2 $2_{1}$ degeneracy 
occur at the point,

$$
\left(\gamma^{*}, \tau^{*}\right)=(14.88647,16.10751)
$$

and a double zero eigenvalue bifurcation occurs at the point

$$
\left(\gamma^{*}, \tau^{*}\right)=(0.01310,1.40245), \quad\left(\gamma^{*}, \tau^{*}\right)=(3.89568,4.95028),
$$

\subsection{Large residence time approximations}

It is useful to study the stability of the system at large residence times since in a such system with a biochemical reaction, the no-washout solution is unstable at large residence time and the periodic solution may occur. For this purpose, we obtain the asymptotic formula, at large values of the residence time,

$$
\begin{aligned}
S_{1}^{*} & \approx \frac{\alpha^{*}}{\tau^{*}}+O\left(\frac{1}{\tau^{*^{2}}}\right), \\
X_{1}^{*} & \approx \frac{\alpha^{*}}{\beta}+\frac{\left(\gamma^{*}-\alpha^{*}\right) \alpha^{*}}{\tau^{*} \beta}+O\left(\frac{1}{\tau^{*^{2}}}\right),
\end{aligned}
$$

To study the stability of the solution at large of the residence time, we substitute equations (43 and 44) onto the Jacobian matrix. We obtain,

$$
\begin{aligned}
J_{11} & =-\frac{\tau^{*}+\alpha-2\left(\gamma^{*}+\beta\right)}{\alpha \tau^{*}}, \quad J_{12}=0, \\
J_{21} & =\frac{\tau^{*}-2 \beta}{\tau^{*}}, \quad J_{22}=-\frac{\beta}{\tau^{*}}, \\
\operatorname{Trace}(\mathrm{J}) & =-\frac{1}{\alpha^{*}}+\frac{-2\left(\gamma^{*}+\beta\right)+\alpha^{*}(1+\beta)}{\alpha^{*} \tau^{*}}, \\
\operatorname{det}(\mathrm{J}) & =\frac{\beta}{\alpha^{*} \tau^{*}}>0 .
\end{aligned}
$$

At sufficiently large of the residence time the second term in equation (45) is approaches zero. This shows that the trace is negative and the determinant is positive. Thus the steady state solution is stable. 


\section{Conclusion}

We have analysed the behaviour of a bioreactor using a generalized Contois growth model including substrate inhibition and a variable yield coefficient. We consider a generalized bioreactor model which includes as special cases the continuous flow and membrane reactors.

The steady-state solutions of the non-dimensional model were found and their stability determined as a function of the residence time. The residence time, at which the washout is stable, satisfies the inequality,

$$
\tau^{*}<\beta(1+e)
$$

This shows that substrate inhibition (e) increases the residence time at washout.

The analysis showed that the system may have up to three steady state solutions and revealed six physically meaningful regions having five distinct steady state diagrams (a, b, c, d, f). In regions (c, d and $\mathrm{f}$ ) of the steady state diagram, a physically meaningful limit point was observed. In regions (b and c) two Hopf points were observed whilst in the last region (d), one Hopf point was observed. The existence of Hopf and limit points is not possible in regions (a, g).

The inclusion of substrate inhibition in the Contois Model equation leads to a critical value of the influent concentration above which the reactor would exhibit the bistability over a region of the residence time, i.e. the no washout solution and the washout solution are both stable at the same value of the residence time. On the other hand if the influent concentration is below the critical value then no bistability occur on the reactor. Consequently, heavy wastewater is more likely to cause bistability.

Furthermore, the inclusion of a variable substrate yield allows the prediction of possible periodic behaviour. Both bistable and periodic behaviour are often undesirable in practice. Our analysis identifies the regions in parameter space where these undesirable phenomena occur and explained how it depends upon the influent concentration. Thus by using an appropriate influent concentration the regions of undesirable behaviour can be avoided in a bioreactor 


\section{A List of Symbols}

\section{Symbol Units Description}

$F \quad \mathrm{dm}^{3} \mathrm{day}^{-1}$ The flow rate through the reactor cascade.

$K_{d} \quad$ day $^{-1} \quad$ The death coefficient.

$K_{d}^{*} \quad$ The dimensionless death rate $\left[K_{d}^{*}=K_{d} / \mu_{\max }\right]$.

$K_{s} \quad|X||S|^{-1} \quad$ The saturation constant.

$S \quad|S| \quad$ The substrate concentration within the reactor .

$S^{*} \quad$ - $\quad$ The dimensionless substrate concentration within the reactor $\left[S^{*}=S / S_{0}\right]$.

$S_{0} \quad|S| \quad$ The concentration of substrate flowing into the reactor.

$V \quad \mathrm{dm}^{3} \quad$ The volume of the reactor in the cascade.

$X \quad|X| \quad$ The microorganisms concentration within the reactor .

$X^{*} \quad$ - $\quad$ The dimensionless microorganisms concentration within the reactor $\left[X^{*}=K_{s} X / S_{0}\right]$.

$X_{0} \quad|X| \quad$ The concentration of the microorganisms flowing into the first reactor the cascade.

$X_{0}^{*} \quad$ - $\quad$ The dimensionless concentration of the microorganisms flowing into the reactor $\left[X_{0}^{*}=K_{s} X_{0} / S_{0}\right]$.

$t$ day The time.

$t^{*} \quad$ - $\quad$ The dimensionless time $\left[t^{*}=\mu_{\max } t\right]$.

$\alpha \quad|X||S|^{-1} \quad$ The yield factor.

$\alpha^{*} \quad$ - $\quad$ The dimensionless yield factor $\left[\alpha^{*}=K_{s} \alpha\right]$.

$\mu(S, X) \quad$ day $^{-1} \quad$ The specific growth rate model.

$\mu_{\max } \quad$ day $^{-1} \quad$ The maximum specific growth rate.

$\tau \quad$ day The residence time.

$\tau^{*} \quad$ - $\quad$ The dimensionless residence time $\left[\tau^{*}=n V \mu_{\max } / F\right]$.

$\beta \quad$ - $\quad$ Reactor parameter model.

$K_{I} \quad|S| \quad$ The inhibition coefficient.

e - $\quad$ The dimensionless inhibition coefficient $\left[e=S_{0} / K_{I}\right]$.

$\gamma \quad|X||S|^{-2} \quad$ The constant in the yield coefficient.

$\gamma^{*} \quad$ - $\quad$ The dimensionless constant in the yield coefficient $\left[\gamma^{*}=\right.$ $\left.\gamma K_{s} S_{0}\right]$ 


\section{B Analysis of the quadratic equation}

We have

$$
a{\widehat{S_{i}^{*}}}^{2}+b{\widehat{S_{i}^{*}}}^{*} c=0
$$

The coefficients $\mathrm{a}, \mathrm{b}$, and $\mathrm{c}$ are given by

$$
a=e \beta-\gamma^{*}, \quad b=-\left(\tau^{*}+\alpha^{*}-\beta\right)+\gamma^{*}, \quad c=\alpha^{*} .
$$

We have the following restriction on parameter values: $e>0, \gamma^{*}>0,0 \leq \beta \leq 1, \tau^{*}>0$. The coefficient c strictly positive. We consider the case $a>0$, which happen when,

$$
\gamma^{*}<e \beta
$$

The coefficients a and $c$, are strictly positive. If $b \geq 0$, then the roots of equation (47), should they are exist, are both non-positive. Conversely, if $b<0$, then the roots of equation (47), should they are exist, are both positive. Thus, a necessary condition for positive solutions of equation (47) is,

$$
b<0 \Rightarrow \tau^{*}>\left(\beta+\gamma^{*}-\alpha^{*}\right)=\tau_{\min , 1}^{*}
$$

We now turn out attention to the discriminant $(\Delta)$ of equation $(47)$. We have

$$
\Delta=b^{2}-4 a c=\left(\tau^{*}-\tau_{1}^{*}\right)\left(\tau^{*}-\tau_{2}^{*}\right)
$$

where

$$
\tau_{1}^{*}=\left(\beta+\gamma^{*}-\alpha^{*}\right)+2 \sqrt{\Phi}, \quad \tau_{2}^{*}=\left(\beta+\gamma^{*}-\alpha^{*}\right)-2 \sqrt{\Phi}, \quad \Phi=\alpha^{*}\left(e \beta-\gamma^{*}\right)>0 .
$$

When $\Delta>0, \Delta=0$, and $\Delta<0$ then equation (47) has two, one and no real solutions respectively. When the roots of the discriminant are real they are labelled $\tau_{1}^{*}$ and $\tau_{2}^{*}$ with $\tau_{1}^{*}>\tau_{2}^{*}$. 
For what follows it is useful to note that when the roots of the discriminant are real we have

$$
\begin{aligned}
& \tau_{1}^{*}>\left(\beta+\gamma^{*}-\alpha^{*}\right)=\tau_{\min , 1}^{*}, \\
& \tau_{2}^{*}<\left(\beta+\gamma^{*}-\alpha^{*}\right)=\tau_{\min , 1}^{*} .
\end{aligned}
$$

Recall that the condition $b<0$ requires $\tau^{*}>\tau_{\min , 1}^{*}$. Hence when $\tau^{*}>\tau_{1}^{*}$ the solutions of equation (47) are both positive and may be physically meaningful. There is a limit point at $\tau^{*}=\tau_{1}^{*}$. The solutions corresponding to the case $\tau^{*}<\tau_{2}^{*}$ are not of interest because they are negative as $b>0$.

\section{Stability analysis for the case $a=0$.}

Using the particular solution (15) for this case, we obtain,

$$
\operatorname{det}(\mathrm{J})=\frac{\beta\left(\tau_{\mathrm{BR}}^{*}-\tau^{*}\right)\left(\tau_{\mathrm{BR}}^{*}-\tau^{*}-\alpha^{*}\right)}{\alpha^{*} \tau^{* 3}}
$$

From the condition for the solution (15) to be physically meaningful, it follows that the determinant (53) is positive and as a result a double zero eigenvalue degeneracy does not occur for case (2).

From equation (53), the first condition of a Hopf bifurcation to occur is satisfied. The conditions $(33,36$ and 37$)$ are solved numerically. Using $\alpha^{*}=0.01$ and $\beta=1$, the location of the double-hopf bifurcation is given by,

$$
\left(\gamma^{*}, \tau^{*}\right)=(0.02254,1.06985)
$$

Figure (5) is an unfolding diagram for the Hopf bifurcation points in the $\gamma^{*}-\tau^{*}$ plane. This figure shows the values of the residence time at which a Hopf bifurcation point occurs as a function of $\gamma^{*}$. It can be observed that there are always two Hopf bifurcation points for $\gamma^{*}>0.02254$ as there is only one $\mathrm{H} 2_{1}$ degeneracy given by equation (54). 


\section{Analysis of $\Delta+b \sqrt{\Delta}$.}

In the case of physically meaningful solution $(\Delta \geq 0)$, we study the behavior of function $(G=$ $\Delta+b \sqrt{\Delta})$. The function $G$ is equal to zero when $\Delta=0$, i.e $\tau^{*}=\tau_{1}^{*}$. It is a clearly positive when $b \geq 0$. From now on we assume that $b<0$. After some algebra the function $G$ can be written in the form,

$$
\begin{aligned}
G=\Delta+b \sqrt{\Delta} & =\frac{-4 \alpha^{*}\left(e \beta-\gamma^{*}\right)}{1-b \sqrt{1 / \Delta}}, \quad \Delta \neq 0, \\
& =\left\{\begin{array}{ll}
\text { negative } & \text { if }\left(e \beta-\gamma^{*}\right)>0, \\
\text { positive } & \text { if }\left(e \beta-\gamma^{*}\right)<0,
\end{array} \text { (Case 3). } .\right.
\end{aligned}
$$




\section{References}

Abdurahman, N., Rosli, Y., \& Azhari, N. (2011a). Development of a membrane anaerobic system (mas) for palm oil mill effluent (pome) treatment. Desalination, 266 (1), 208-212.

Abdurahman, N., Rosli, Y., Azhari, N., \& Tam, S. (2011). Biomethanation of palm oil mill effluent (pome) by membrane anaerobic system (mas) using pome as a substrate. World Academy of Science, Engineering and Technology, 75, 419-424.

Abrams, P. A., \& Ginzburg, L. R. (2000). The nature of predation: prey dependent, ratio dependent or neither? Trends in Ecology 6 Evolution, 15(8), 337-341.

Ajbar, A. (2010). Study of the operability of nonideal continuous bioreactors. Chemical Engineering Communications, 198(3), 385-415.

Ajbar, A. (2012). Study of complex dynamics in pure and simple microbial competition. Chemical Engineering Science, 80, 188 - 194.

Ajbar, A., AlAhmad, M., \& Ali, E. (2011). On the dynamics of biodegradation of wastewater in aerated continuous bioreactors. Mathematical and Computer Modelling, 54(9), 1930-1942.

Ajbar, A., \& Alhumaizi, K. (2011). Dynamics of the chemostat: A bifurcation theory approach. CRC Press.

Alqahtani, R. T., Nelson, M. I., \& Worthy, A. L. (2012). Analysis of a chemostat model with variable yield coefficient: Contois kinetics. ANZIAM Journal, 53, C155-C171.

Andrews, J. F. (1968). A mathematical model for the continuous culture of microorganisms utilizing inhibitory substrates. Biotechnology and Bioengineering, 10(6), 707-723.

Ardestani, F. (2012). Survey of the nutrient utilization and cell growth kinetic with verhulst, contois and exponential models for penicillium brevicompactum atcc 16024 in batch bioreactor. World Applied Sciences Journal, 16(1), 135-140.

Bongochgetsakul, N., \& Ishida, T. (2008). A new analytical approach to optimizing the design of large-scale composting systems. Bioresource technology, 99(6), 1630-1641. 
Cabrera, F., \& Maria, I. (2011). Deterministic approach to the study of the interaction predator-prey in a chemostat with predator mutual interference. implications for the paradox of enrichment. Ecological Modelling, 222(3), 598-605.

Contois, D. (1959). Kinetics of bacterial growth: relationship between population density and specific growth rate of continuous cultures. Journal of General Microbiology, 21 (1), 40-50.

Crooke, P. S., Wei, C.-J., \& Tanner, R. D. (1980). The effect of the specific growth rate and yield expressions on the existence of oscillatory behavior of a continuous fermentation model. Chemical Engineering Communications, 6(6), 333-347.

Czeczot, J., Metzger, M., Babary, J.-P., \& Nihtilä, M. (2000). Filtering in adaptive control of distributed parameter bioreactors in the presence of noisy measurements. Simulation Practice and Theory, 8(1), 39-56.

Dorofeev, A., Glagolev, M., Bondarenko, T., \& Panikov, N. (1992). Observation and explanation of the unusual growth-kinetics of arthrobacter-globiformis. Microbiology, 61(1), $24-31$.

Emerald, F., Eljeeva, M., Prasad, D. S., Ravindra, M. R., \& Pushpadass, H. A. (2012). Performance and biomass kinetics of activated sludge system treating dairy wastewater. International Journal of Dairy Technology, 65(4), 609-615.

Ermentrout, B. (2002). Simulating, analyzing, and animating dynamical systems: a guide to xppaut for researchers and students (Vol. 14). Siam.

Gawande, N. A., Reinhart, D. R., \& Yeh, G.-T. (2010). Modeling microbiological and chemical processes in municipal solid waste bioreactor, part i: Development of a three-phase numerical model biokemod-3p. Waste management, 30(2), 202-210.

Goudar, C. T., Ganji, S. H., Pujar, B. G., \& Strevett, K. A. (2000). Substrate inhibition kinetics of phenol biodegradation. Water environment research, 72(1), 50-55.

Goudar, C. T., \& Strevett, K. A. (1998). Estimating growth kinetics of penicillium chrysogenum by nonlinear regression. Biochemical engineering journal, 1(3), 191-199. 
Grady, C., \& Lim, H. (1999). Biological wastewater treatment. New York and Basel: Marcel Dekker, Inc.

Gray, B., \& Roberts, M. (1988). A method for the complete qualitative analysis of two coupled ordinary differential equations dependent on three parameters. Proceedings of the Royal Society of London. A. Mathematical and Physical Sciences, 416(1851), 361-389.

Guerrero, L., Montalvo, S., Coronado, E., Chamy, R., Poirrier, P., Crutchik, D., ... Borja, R. (2009). Performance evaluation of a two-phase anaerobic digestion process of synthetic domestic wastewater at ambient temperature. Journal of Environmental Science and Health Part A, 44(7), 673-681.

Harmand, J., \& Godon, J. (2007). Density-dependent kinetics models for a simple description of complex phenomena in macroscopic mass-balance modeling of bioreactors. Ecological modelling, 200(3), 393-402.

Hemsi, P., Shackelford, C. D., \& Figueroa, L. A. (2010). Calibration of reactive transport models for remediation of mine drainage in solid-substrate biocolumns. Journal of Environmental Engineering, 136(9), 914-925.

Hernalsteens, S., \& Maugert, F. (2010). Synthesis of fructooligosaccharides using extracellular enzymes from rhodotorula sp. Journal of Food Biochemistry, 34(3), 520 -534.

Hidaka, T., Horie, T., Akao, S., \& Tsuno, H. (2010). Kinetic model of thermophilic l-lactate fermentation by bacillus coagulans combined with real-time PCR quantification. Water research, $44(8), 2554-2562$.

Hu, W., Thayanithy, K., \& Forster, C. (2002). A kinetic study of the anaerobic digestion of ice-cream wastewater. Process Biochemistry, 37(9), 965-971.

Huang, X., \& Zhu, L. (2005). A three dimensional chemostat with quadratic yields. Journal of mathematical chemistry, 38(4), 575-588.

Huang, X., \& Zhu, L. (2009). Bifurcation in the stable manifold of the bioreactor with nth and mth th order polynomial yields. Journal of Mathematical Chemistry, 46, 199-213. 
Ilichev, V. (2008). Active and passive states in ecological models (stabilization, adaptation, and survivability). Journal of Computer and Systems Sciences International, 47(5), 830-839.

Işik, M., \& Sponza, D. T. (2005). Substrate removal kinetics in an upflow anaerobic sludge blanket reactor decolorising simulated textile wastewater. Process Biochemistry, 40 (3), 11891198.

Lee, I. H., Fredrickson, A., \& Tsuchiya, H. (1976). Damped oscillations in continuous culture of lactobacillus plantarum. Journal of General Microbiology, 93(2), 204-208.

Mazutti, M. A., Corazza, M. L., Maugeri Filho, F., Rodrigues, M. I., Corazza, F. C., \& Treichel, H. (2009). Inulinase production in a batch bioreactor using agroindustrial residues as the substrate: experimental data and modeling. Bioprocess and biosystems engineering, 32(1), 85-95.

Michael, L., \& Fikret, K. (2002). Bioprocess engineering: Basic concepts. Prentice-Hall International, USA.

Monod, J. (1949). The growth of bacterial cultures. Annual Reviews in Microbiology, 3(1), 371-394.

Moser, H. (1958). The dynamics of bacterial populations maintained in the chemostat. Carnegie Institution of Washington.

Nelson, M., Balakrishnan, E., Sidhu, H., \& Chen, X. (2008b). A fundamental analysis of continuous flow bioreactor models and membrane reactor models to process industrial wastewaters. Chemical Engineering Journal, 140(1), 521-528.

Nelson, M., Chen, X., \& Sidhu, H. (2008a). Reducing the emission of pollutants in industrial wastewater through the use of membrane bioreactors. In R. Hosking \& E. Venturino (Eds.), Aspects of mathematical modelling (p. 95-107).

Nelson, M., \& Sidhu, H. (2007). Reducing the emission of pollutants in food processing wastewaters. Chemical Engineering and Processing: Process Intensification, 46(5), 429-436.

Nelson, M., \& Sidhu, H. (2009). Analysis of a chemostat model with variable yield coefficient: Tessier kinetics. Journal of mathematical chemistry, 46(2), 303-321. 
Pinna, A., Lallai, A., Mura, G., \& Grosso, M. (2009). Comparison across different models for the description of batch biodegradation processes. Chemical Engineering Transactions, $1227-1232$.

Poh, P., \& Chong, M. (2010). Biomethanation of palm oil mill effluent (pome) with a thermophilic mixed culture cultivated using pome as a substrate. Chemical Engineering Journal, $164(1), 146-154$.

Ramirez, I., Mottet, A., Carrère, H., Déléris, S., Vedrenne, F., \& Steyer, J.-P. (2009). Modified adm1 disintegration/hydrolysis structures for modeling batch thermophilic anaerobic digestion of thermally pretreated waste activated sludge. Water research, 43(14), 3479-3492.

Rozich, A., \& Gaudy Jr, A. (1985). Response of phenol-acclimated activated sludge process to quantitative shock loading. Journal of Water Pollution Control Federation, 795-804.

Sari, T. (2010a). Comments on limit cycles in the chemostat with constant yields mathematical and computer modelling 45 (2007) 927-932. Mathematical and Computer Modelling, 52(9), $1822-1824$.

Sari, T. (2010b). A lyapunov function for the chemostat with variable yields. Comptes Rendus Mathematique, 348(13), 747-751.

Sokol, W. (1987). Oxidation of an inhibitory substrate by washed cells (oxidation of phenol by pseudomonas putida). Biotechnology and bioengineering, 30(8), 921-927.

Song, G., \& Li, X. (1999). Stability of solution to the chemostat system with non-constant consuming rate. J. Biomath, 14(1), 24-27.

Sun, S., Wu, B., Zhao, D., Zhang, X., Zhang, Y., Li, W., \& Cheng, S. (2009). Optimization of xhhh strain biodegradation with metal ions for pharmaceautical wastewater treatment. Journal Of Environmental Biology / Academy Of Environmental Biology, 30, 877 - 882.

Tan, Y., Wang, Z.-X., \& Marshall, K. C. (1996). Modeling substrate inhibition of microbial growth. Biotechnology and bioengineering, 52(5), 602-608.

Tang, W.-T., \& Fan, L.-S. (1987). Steady state phenol degradation in a draft-tube, gas-liquidsolid fluidized-bed bioreactor. AIChE journal, 33(2), 239-249. 
Tessier, G. (1936). Les lois quantitatives de la croissance. Annales de Physiologie et de Physiochimie Biologique, 12, 527-573.

Wolkowicz, G. S., \& Lu, Z. (1992). Global dynamics of a mathematical model of competition in the chemostat: general response functions and differential death rates. SIAM Journal on Applied Mathematics, 52(1), 222-233.

Zhang, J., Shao, X., Townsend, O. V., \& Lynd, L. R. (2009). Simultaneous saccharification and co-fermentation of paper sludge to ethanol by saccharomyces cerevisiae rwb222part i: Kinetic modeling and parameters. Biotechnology and bioengineering, 104(5), 920-931.

Zhou, H., Lu, W., Wen, J., \& Ma, L. (2009). Kinetic analysis of 11a-hydroxylation of steroids by rhizopus nigricans. Journal of Molecular Catalysis B: Enzymatic, 56, 136 - 141.

Zhu, L. (2007). Limit cycles in chemostat with constant yields. Mathematical and computer modelling, 45(7), 927-932. 


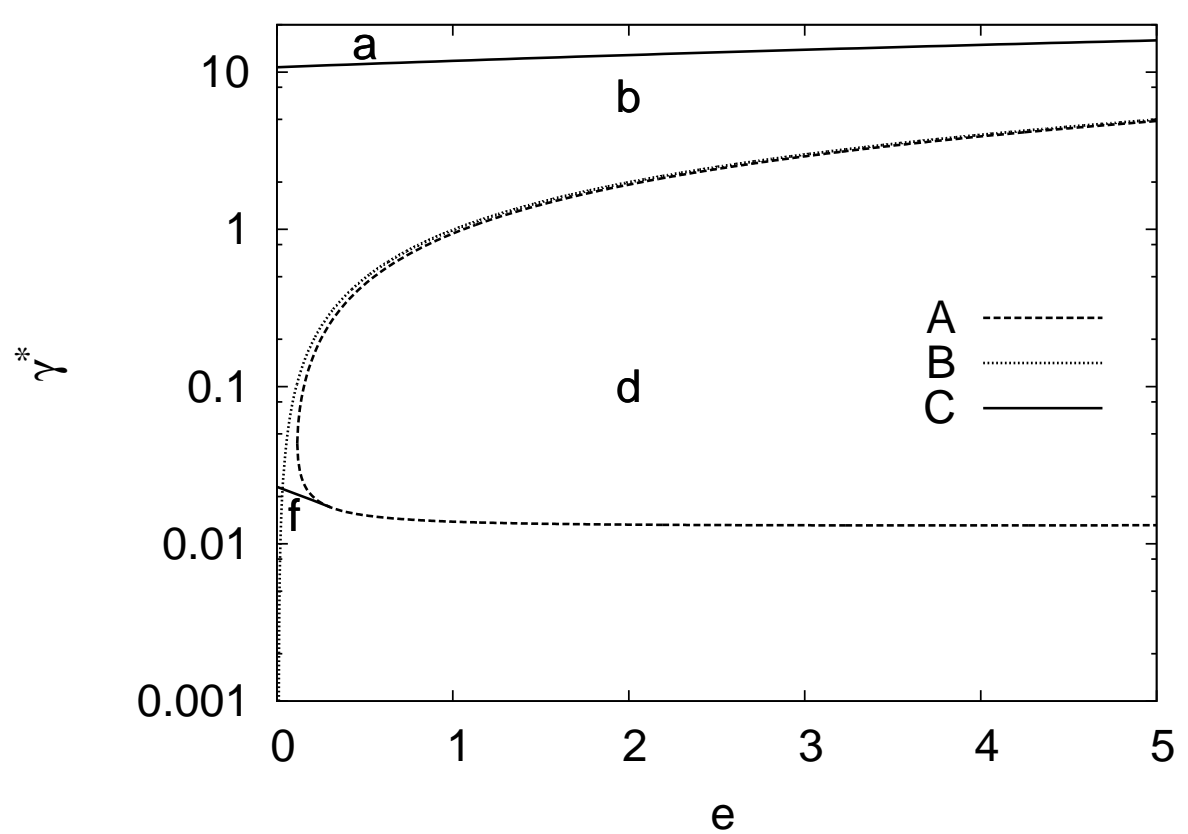

(a)

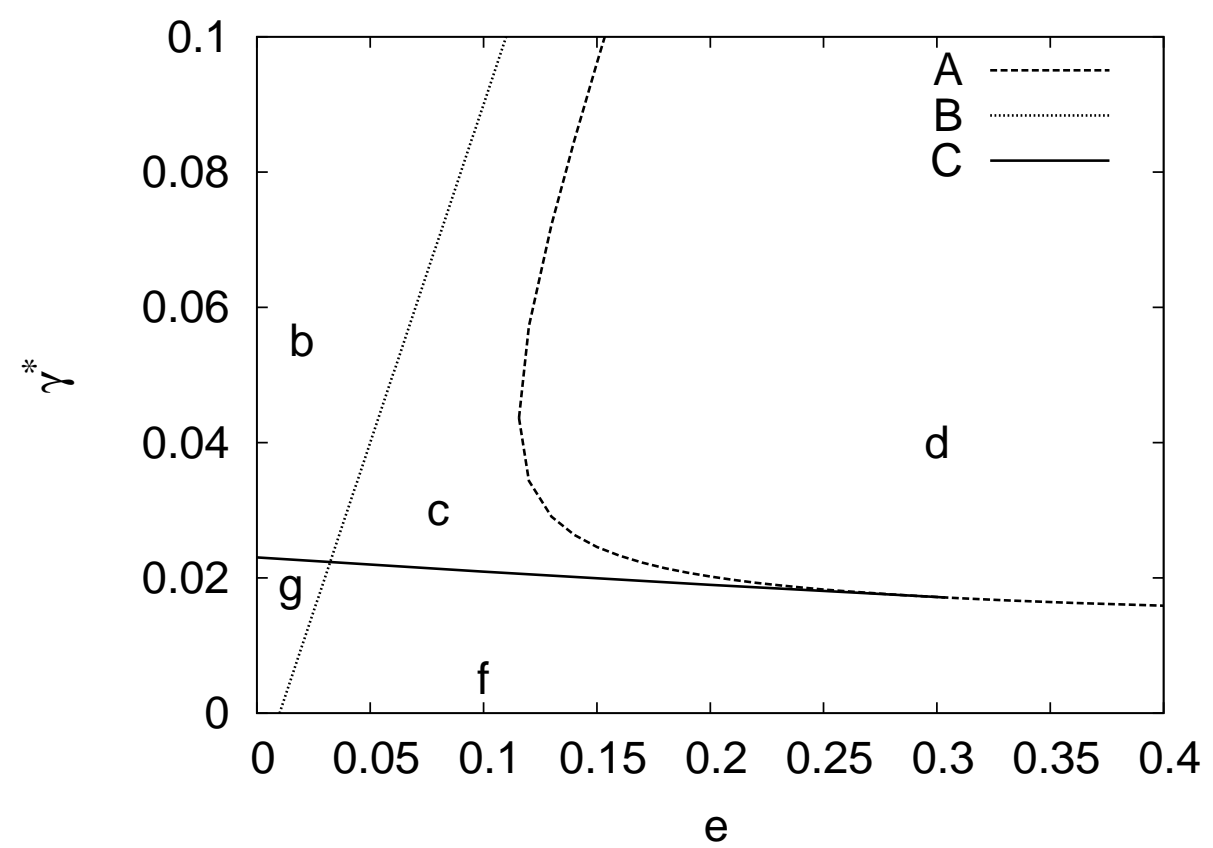

(b) Small area from Figure 2(a)

Figure 2: Bifurcation diagram. Line (A) is the double-zero eigenvalue locus, Line (B) corresponds to the case $\gamma^{*}=\beta e-\alpha^{*}$ locus. Line $(\mathrm{C})$ represents the $\mathrm{H} 2_{1}$ locus. The value of parameters: $\beta=1$ and $\alpha^{*}=0.01$. 


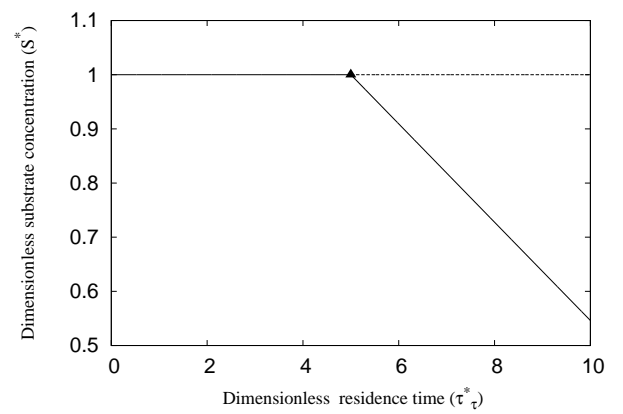

(a) Region (a) $\left(e, \gamma^{*}\right)=(4,15)$

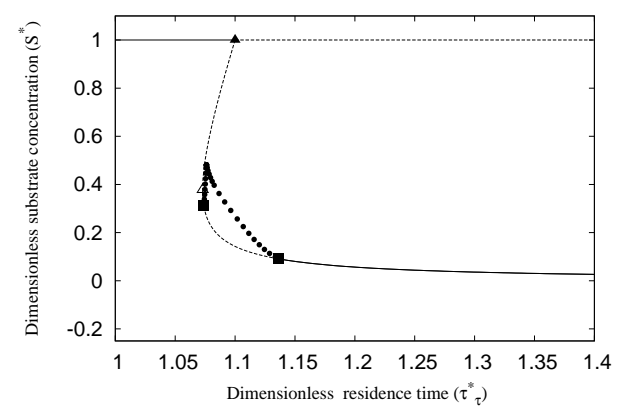

(c) Region (c) $\left(e, \gamma^{*}\right)=(0.1,0.03)$

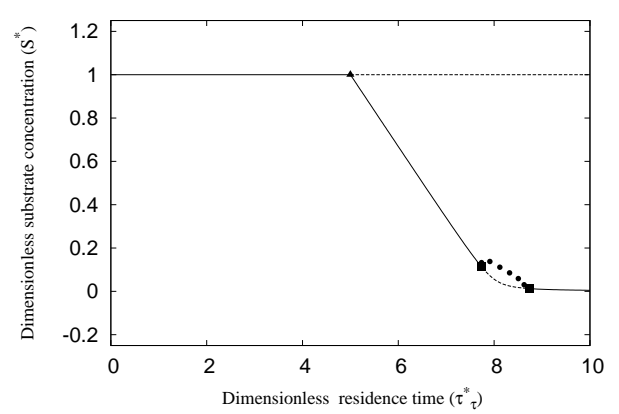

(b) Region (b) $\left(e, \gamma^{*}\right)=(4,7)$

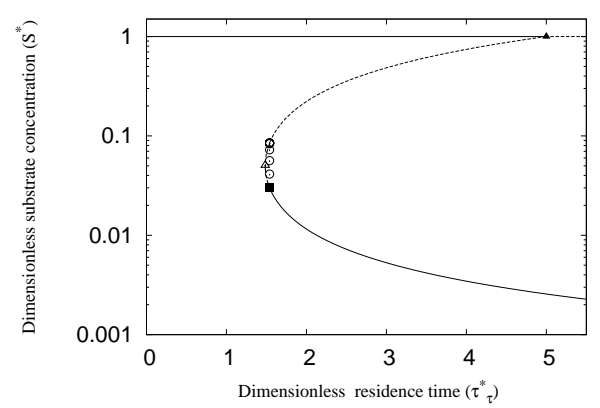

(d) Region (d) $\left(e, \gamma^{*}\right)=(4,0.1)$

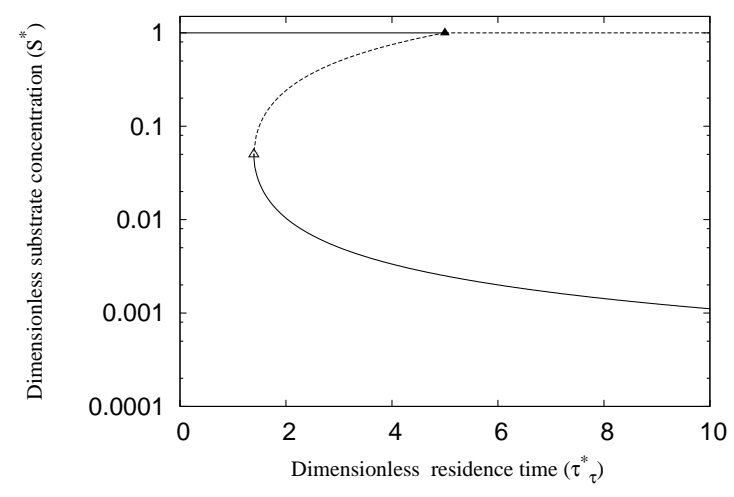

(e) Region (f) $\left(e, \gamma^{*}\right)=(4,0.005)$

Figure 3: Diagram showing the different steady state regions of Figure (2). $\beta=1$ and $\alpha^{*}=0.01$. All the symbols are explained in section (4). 


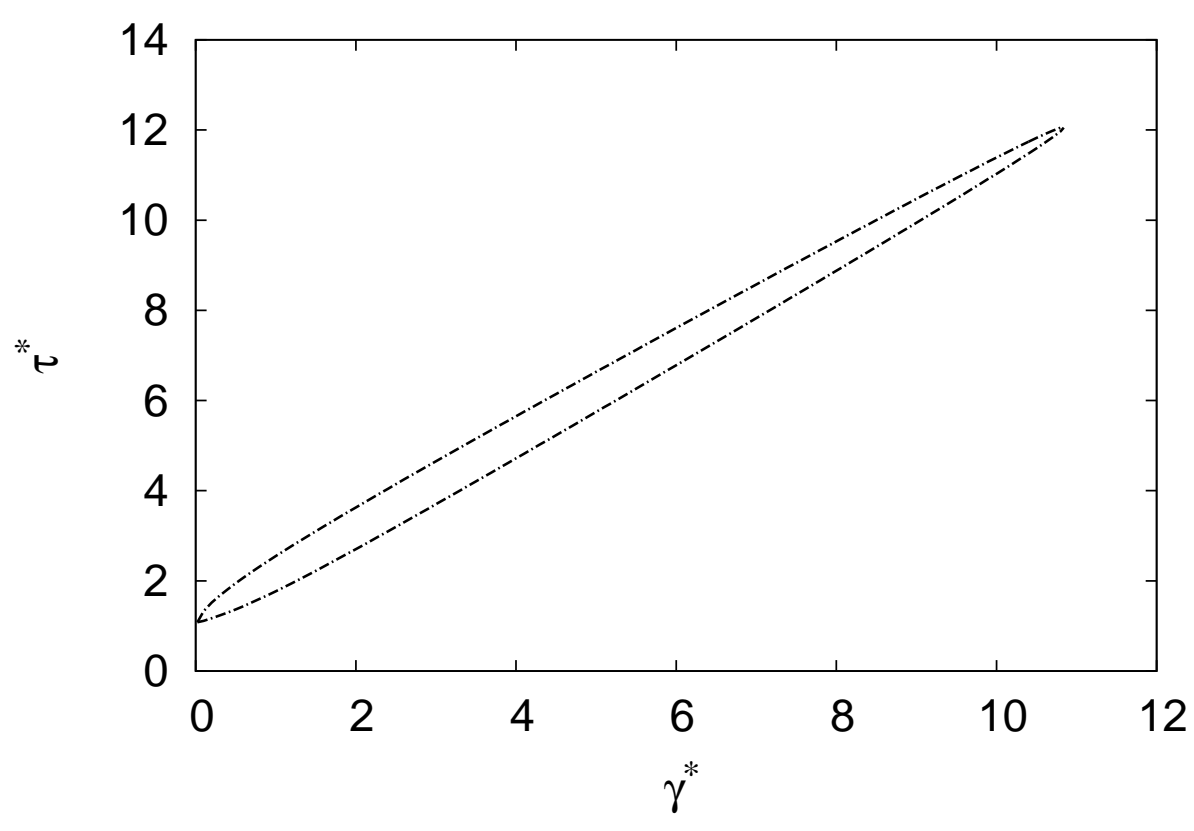

(a) $\mathrm{e}=0.1$

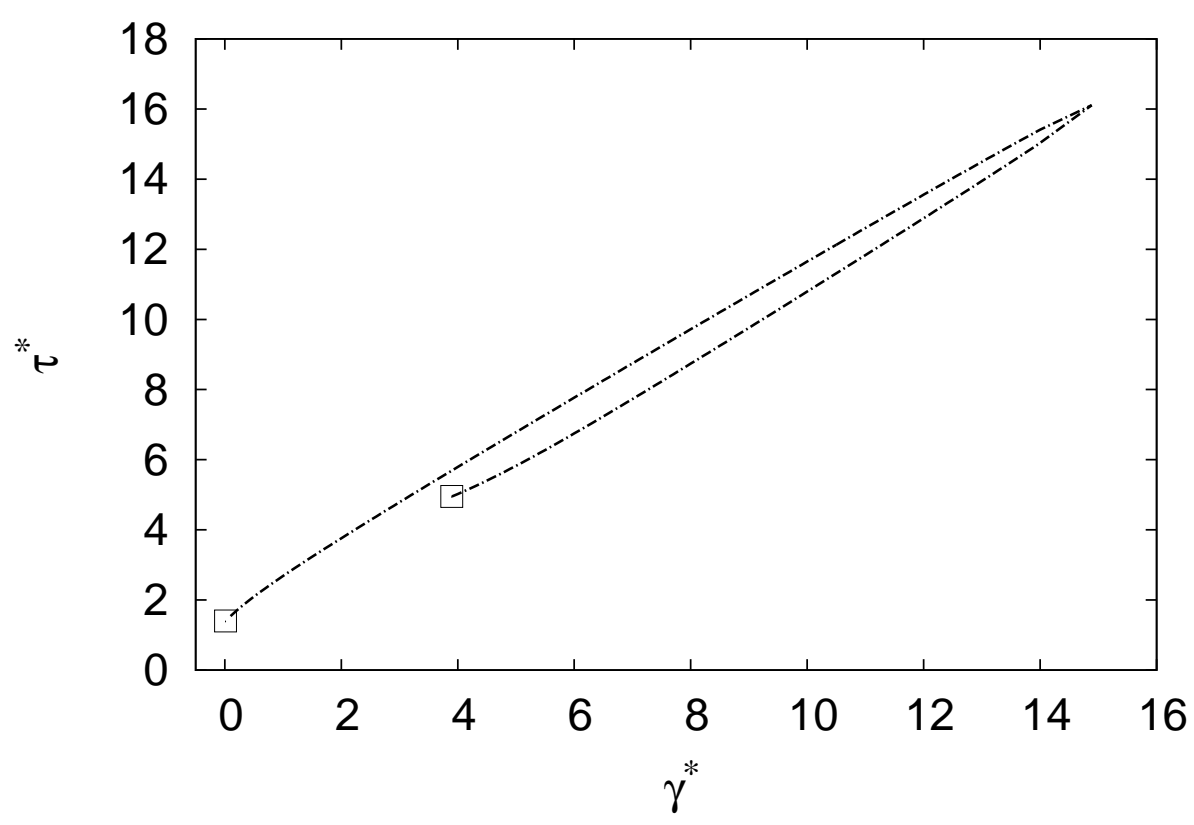

(b) $\mathrm{e}=4$

Figure 4: Unfolding diagram for a single reactor showing the Hopf bifurcation locus. Parameter values: $\beta=1, \alpha^{*}=0.01$. All the symbols are explained in section (4). 


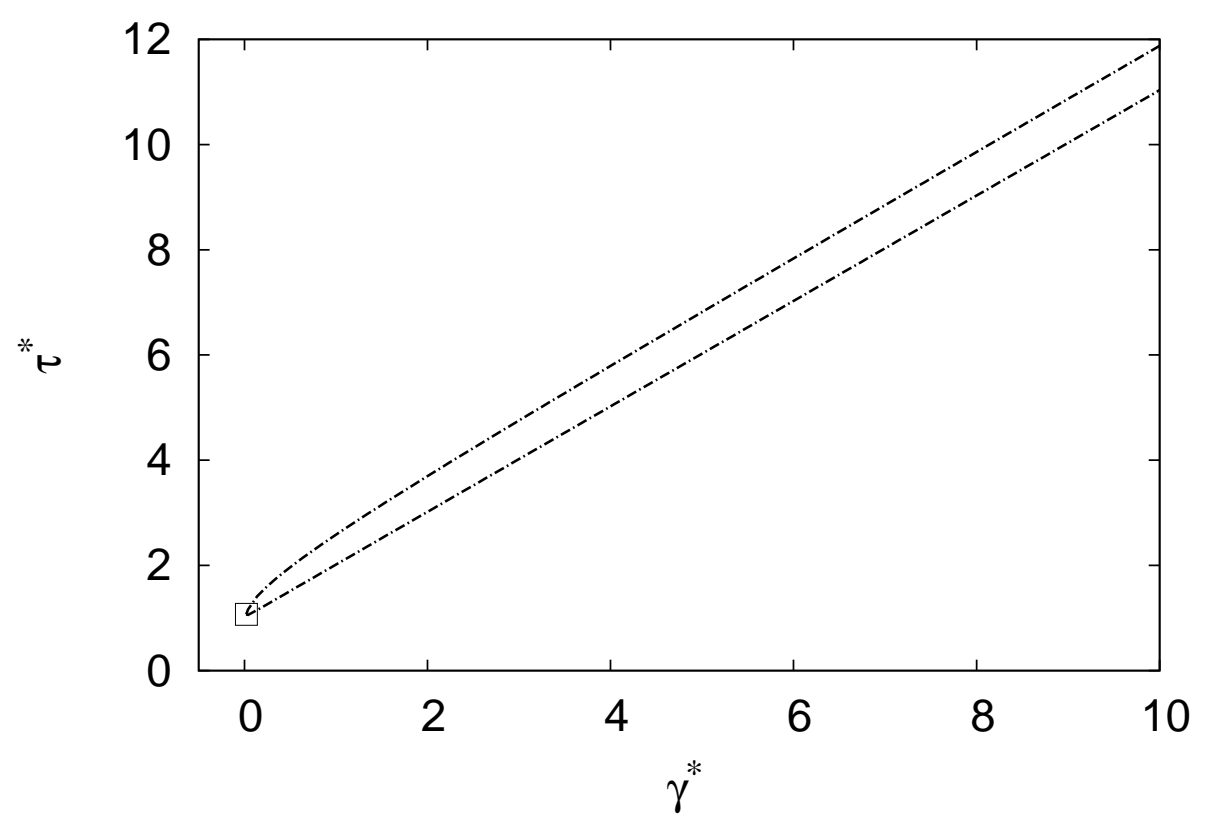

Figure 5: Unfolding diagram for case $(2)(a=0)$ showing the Hopf bifurcation locus. Parameter values: $\beta=1, \alpha^{*}=0.01$. All the symbols are explained in section (4). 